\title{
Reactor para tratamiento de aguas residuales por fotocatálisis heterogénea
}

\author{
B.J. Lorenzano Hernández ${ }^{1 *}$, K.R. Márquez Herrera ${ }^{1}$, R. Yañez Guzmán, \\ M.A. Camacho González¹, F.J. Manríquez Rojas²
}

\begin{abstract}
1División de Procesos Industriales, Nanotecnología área materiales. Universidad Tecnológica de Tecámac, Carr. Fed. México-Pachuca Km. 37.5, Col. Sierra Hermosa, Tecámac, Estado de México, 55740, México 2División Química Biológicas, Química área biotecnología, Universidad Tecnológica de Tecámac, Carr. Fed. México-Pachuca Km. 37.5, Col. Sierra Hermosa, Tecámac, Estado de México, 55740, México

*Autor de correspondencia: brayanjlhz2@gmail.com
\end{abstract}

\section{RESUMEN}

Se construyó un reactor vertical de vidrio con aireación ascendente a escala de los empleados en las estaciones de depuración de aguas residuales, EDAR, cuyas condiciones permiten reducir la concentración de contaminantes orgánicos presentes en agua residual de origen industrial y núcleos poblacionales, por fotocatálisis heterogénea. El reactor es provisto de 6 discos perforados de acero inoxidable recubiertos por la técnica de dip-coating revólver, con películas fotocatalíticas del sistema mixto titaniaalúmina sintetizado por la técnica sol-gel; la hidrodinámica al interior del reactor permitió lograr una eficiencia de degradación promedio del $97.43 \%$ para azul de metileno y 98.12 $\%$ para fenol por medición indirecta de reducción de absorbancia a 665 y $220 \mathrm{~nm}$ respectivamente y $96.51 \%$ para agua residual por la medida de la reducción del carbono orgánico total, COT; en un promedio de 6 a 7 horas de reacción. Las películas, se caracterizaron por espectroscopía infrarroja con trasformada de fourier (FT-IR), se observaron las bandas características del enlace Al-O a $569 \mathrm{~cm}^{-1}$, por espectroscopía UV-Vis muestra elevada absorción en el intervalo UV-Vis, de 300 a $700 \mathrm{~nm}$, siendo la máxima a $350 \mathrm{~nm}$; por difracción de rayos X (DRX), se obtuvieron los planos (101), (004), (200), (211), (204), (220) y (215), característicos para la fase anatasa del $\mathrm{TiO}_{2}$ y los planos (220), (311), (400) y (844) correspondientes a una estructura cúbica simple

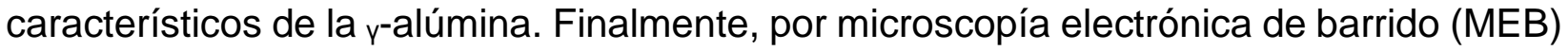
se observó una película homogénea con partículas del orden de 30-40 nm libre de fracturas.

Palabras Clave: Reactor, Contaminantes orgánicos, Sol-Gel, Fotocatálisis heterogénea, Titania, Alúmina.

\section{INTRODUCCIÓN}

En la actualidad, la reducción de contaminantes orgánicos presentes en agua residual de origen industrial y de las redes de alcantarillado de los núcleos de población; se realiza en estaciones de depuración de aguas residuales, EDAR's; en las que la materia orgánica es transformada en biomasa y compuestos orgánicos volátiles por un proceso microbiológico complejo realizado en ausencia de oxígeno. Las EDAR's además de entregar como producto el agua tratada, genera subproductos como gas metano, de alto valor agregado como combustible y fango o lodos activados: el complejo 
microbiológico empleado en la digestión anaerobia, al cumplir su vida útil es desechado como composta una vez inactivado. En términos generales, las EDAR's, son estaciones de tratamiento de agua residual que involucran una gran cantidad de operaciones unitarias, además de largos tiempos de tratamiento, traducido en costos elevados en el proceso de depuración completo [1].

Recientemente, los procesos de oxidación avanzada, PAO's, coadyuvan y/o sustituyen los procesos de digestión anaerobia, reduciendo la cantidad de operaciones realizadas en una EDAR. La fotocatálisis heterogénea, es un proceso avanzado de oxidación fotoquímica, que emplea un material semiconductor de naturaleza cerámica que, a escala nanométrica, presenta propiedades catalíticas. Cuando la superficie del material fotocatalizador es excitada con una longitud de onda equivalente o mayor a su energía de banda prohibida, se forman radicales hidroxilos $\left(\bullet \mathrm{OH}^{-}\right)$y/o superóxido $\left(\bullet \mathrm{O}_{2}{ }^{2-}\right)$ de elevado poder oxidante, los cuales contribuyen a la mineralización total de los contaminantes orgánicos en el agua, por reacciones sucesivas de óxido-reducción, además de causar la muerte por lisis celular de microorganismos patógenos por ruptura de su membrana citoplasmática, debido a la alta concentración de estos radicales [2].

En este trabajo se diseñó y construyó un reactor similar a los de digestión anaerobia en una EDAR, con deflectores recubiertos con una película fotocatalítica de $\mathrm{TiO}_{2}-\mathrm{Al}_{2} \mathrm{O}_{3}$, que al ser excitada con radiación visible y estar en contacto directo con agua contaminada, logra degradar los compuestos orgánicos presentes y además, causa la muerte de microorganismos patógenos que afectan la salud pública. Se espera que esta tecnología innovadora, represente a futuro una oportunidad para mejorar el proceso de recuperación de agua residual a un menor costo a través de la Nanotecnología [3].

\section{PARTE EXPERIMENTAL}

\section{Síntesis del complejo hidroxilado de aluminio y del sistema mixto titania alúmina}

La síntesis se llevó a cabo vía sol gel, en dos etapas consecutivas, en la primera se utilizó una solución precursora $0.1 \mathrm{M}$ en $50 \mathrm{ml}$. de; sulfato de aluminio $\mathrm{Al}_{2}\left(\mathrm{SO}_{4}\right)_{3} \cdot 14 \mathrm{H}_{2} \mathrm{O}$ marca Kemira ALC 2-5 con un $90 \%$ de pureza. Se tituló con $\mathrm{NaOH} 1 \mathrm{M}$ en $100 \mathrm{ml}$ y se aplicó temperatura de $70^{\circ} \mathrm{C}$ con agitación magnética constante, para favorecer la formación de complejos oxo-hidroxilados y la obtención de un precipitado. En la segunda etapa se utilizaron $20 \mathrm{ml}$ de una solución etanólica (1.5 ml TBT: $8.5 \mathrm{ml} \mathrm{Et-OH})$ de terbutóxido de titanio; Ti[OC( $\left.\left(\mathrm{CH}_{3}\right)_{3}\right]_{4}$, TBT; marca sigma aldrich con un $97 \%$ de pureza, se adicionó en $20 \mathrm{ml}$ de agua destilada de $\mathrm{pH}=1.5$ con $\mathrm{HNO}_{3}$ al $20 \%$ v/v que contenía el sólido húmedo del complejo hidroxilado de aluminio. Se mantuvo en agitación vigorosa para obtener una suspensión coloidal lechosa que se utilizó en la inmersión de sustratos de acero inoxidable [4]. 
Deposición de películas del sistema mixto titania-alúmina en discos perforados de acero inoxidable

Se empleó la metodología dip-coating revólver en 6 discos de acero inoxidable [4]. Se sumergieron a una velocidad de $20 \mathrm{~mm} / \mathrm{min}$ en la suspensión coloidal obtenida en el proceso de síntesis. Las películas depositadas se llevaron a dos tratamientos térmicos consecutivos a una temperatura de $80{ }^{\circ} \mathrm{C}$ y $180{ }^{\circ} \mathrm{C}$ respectivamente, para finalmente someter a sinterizado a una temperatura de $700{ }^{\circ} \mathrm{C}$ durante 5 horas. Las películas obtenidas se caracterizaron para determinar la composición química, propiedades ópticas, estructura cristalina y topografía superficial por espectroscopía infrarroja con trasformada de Fourier, FT-IR; espectroscopía ultravioleta visible, UV-Vis; difracción de rayos $\mathrm{X}, \mathrm{DRX}$; y microscopía electrónica de barrido, MEB; respectivamente.

\section{Diseño y construcción de un reactor vertical de aireación ascendente}

Se diseñó un reactor (Figura 1) considerando las dimensiones de escalamiento industrial empleando el software Solid works 2015. Se realizó el montaje estructural para realizar pruebas de funcionamiento general, caracterización de la hidrodinámica al interior del mismo, y corregir detalles.
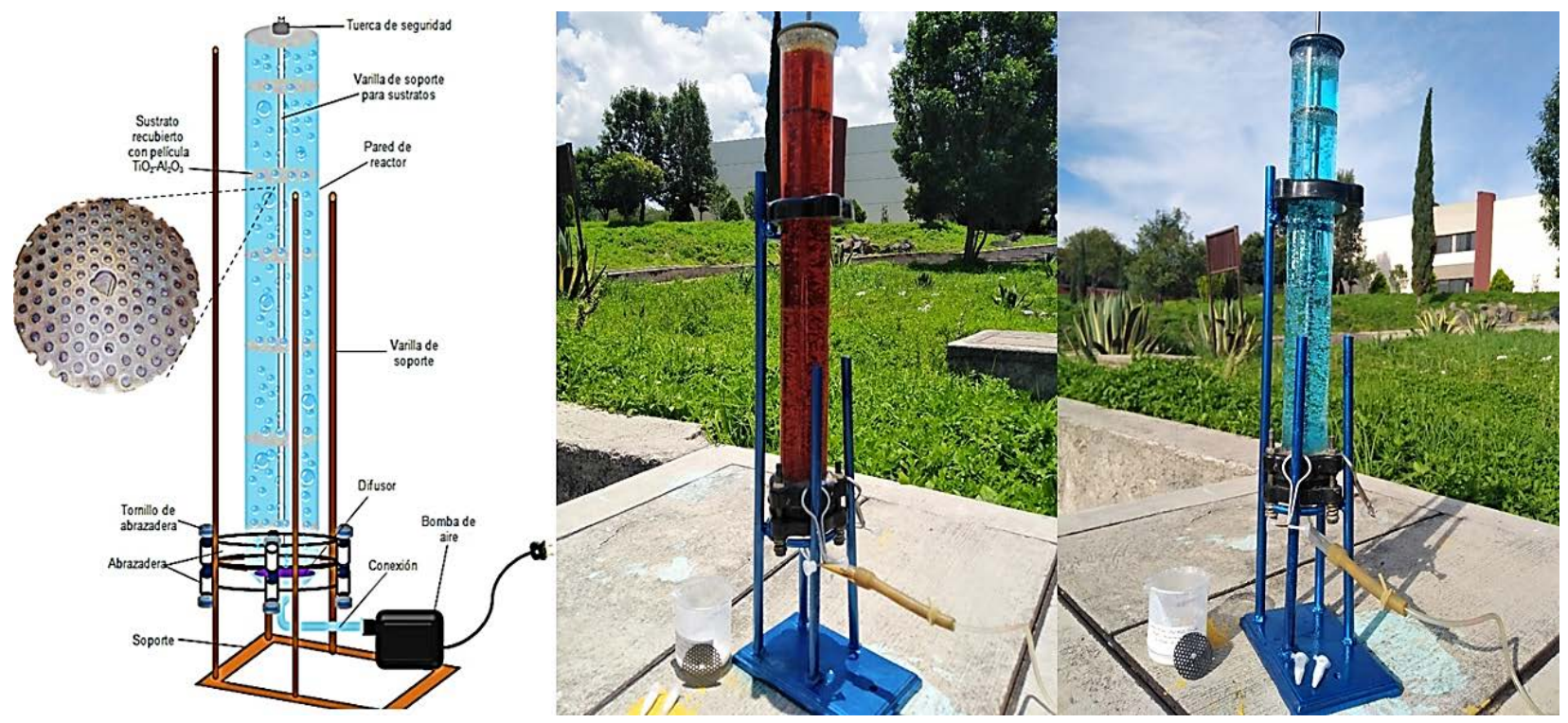

Figura 1. Prototipo y reactor funcional construido en Solidworks 2015, sustrato recubierto con película fotocatalítica de $\mathrm{TiO}_{2}-\mathrm{Al}_{2} \mathrm{O}_{3}$ [Elaboración propia].

\section{Degradación fotocatalítica.}

La Figura 2, muestra las condiciones de reacción para la degradación de azul de metileno, fenol y una muestra de agua residual proveniente de la EDAR ubicada en Av. Rancho Sierra Hermosa, Col. Sierra Hermosa, municipio de Tecámac, Edo. México, México. 


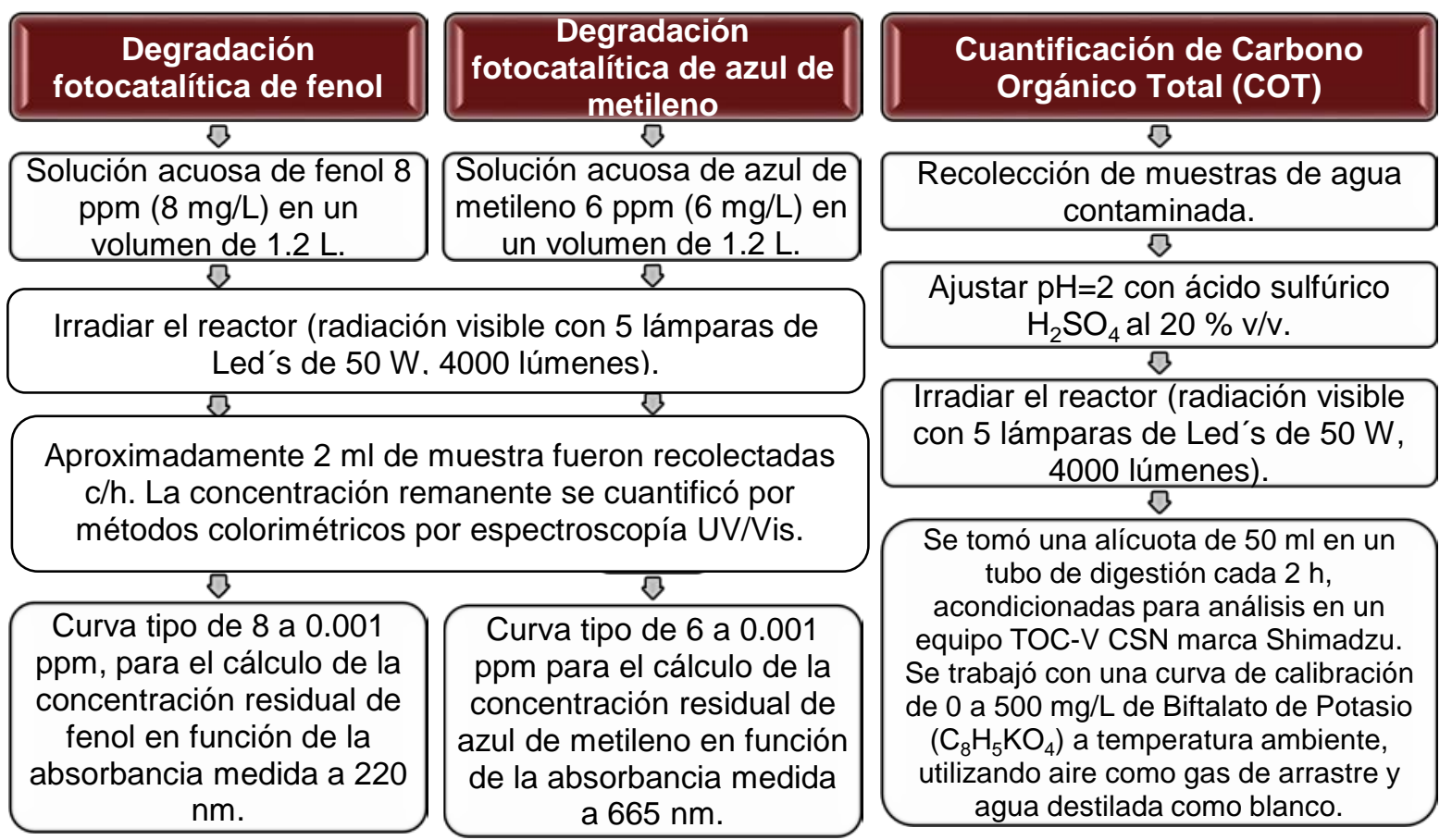

Figura 2. Diagrama general para cuantificar la degradación fotocatalítica de contaminantes orgánicos al interior del reactor [Elaboración propia].

\section{RESULTADOS}

Las películas depositadas se caracterizaron mediante las técnicas de espectroscopía infrarroja con trasformada de fourier (FT-IR), usando un equipo FT-IR marca Perkin Elmer modelo Spectrum two y por espectroscopìa UV-Vis, utilizando un espectrofotómetro marca Perkin Elmer modelo Lambda 35 rango de 200-700 nm del laboratorio de Nanotecnología de la Universidad Tecnológica de Tecámac. Para identificar la forma cristalina se utilizó la técnica de difracción de rayos $X(D R X)$ con un difractómetro marca Bruker modelo ECO D8 Advance. La microscopía electrónica de barrido (MEB) permitió identificar, morfología de las nanopartículas depositadas, tamaño y dispersión homogénea de la película sobre el sustrato, se utilizó un equipo marca JEOL, modelo JSM-10L del Área Académica de Ciencias de la Tierra y Materiales, AACTyM, de la Universidad Autónoma del Estado de Hidalgo, UAEH.

Para la cuantificación de carbono orgánico total se empleó un equipo TOC-V CSN marca Shimadzu con un mostrador automático ASI-V marca Shimadzu de la Universidad Tecnológica de Tecámac. Se trabajó con una curva de calibración de 0 a 500 mg/L de biftalato de potasio $\left(\mathrm{C}_{8} \mathrm{H}_{5} \mathrm{KO}_{4}\right)$. Los gráficos fueron analizados utilizando el software OriginPro Lab 8. 
Espectroscopía infrarroja con trasformada de fourier (FT-IR)

La Figura 3 muestra el espectro de trasmisión infrarroja para el sistema titania-alúmina. Se observa una banda a $3430 \mathrm{~cm}^{-1}$ del enlace $\mathrm{O}-\mathrm{H}$ que indica la presencia de agua, así como la aparición de la banda a $1123 \mathrm{~cm}^{-1}$ relacionada con los iones sulfato $\left(\mathrm{SO}_{4}\right)^{-2}$. La banda que caracteriza el enlace Al-O se presentó a los $569 \mathrm{~cm}^{-1}$. Las vibraciones del enlace Ti-O se reportan en un rango de $480-485 \mathrm{~cm}^{-1}$ [5]. Las limitantes del equipo empleado hacen que ésta vibración no se visualice en el gráfico presentado. Analizando el comportamiento de las gráficas y al proceso de síntesis se puede presumir la presencia del enlace, que podría confirmarse por Espectroscopía Raman.

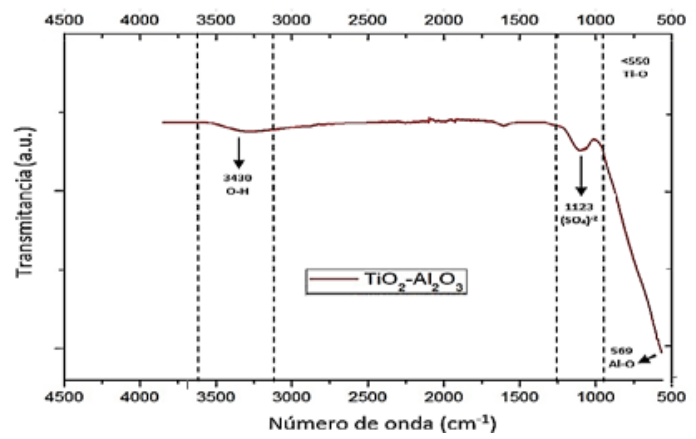

Figura 3. Espectro IR para el sistema de titania-alúmina sintetizados por el método sol-gel.

Espectroscopía UV - Vis

En la Figura 4 se muestra que el sistema mixto de titania-alúmina, sintetizado por la técnica sol-gel, absorbe radiación en el rango comprendido en el UV cercano y visible entre 300 y $700 \mathrm{~nm}$, siendo la máxima absorción alrededor de 340-360 nm, lo que demuestra que los dominios fotocatalíticos propios del $\mathrm{TiO}_{2}$, usualmente entre los 200$220 \mathrm{~nm}$ fueron mejorados al realizar un dopaje con $\mathrm{Al}_{2} \mathrm{O}_{3}$. Por lo que el proceso de fotocatálisis podrá ser llevado a cabo empleando luz solar o una fuente de radiación artificial (LED).

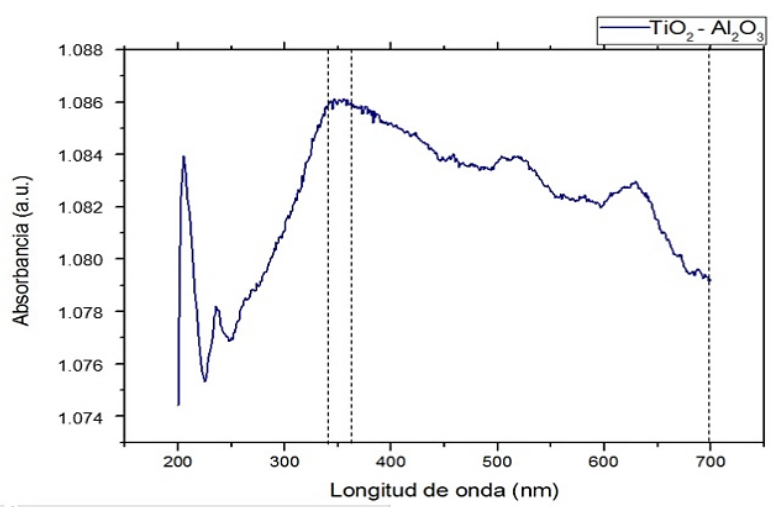

Figura 4. Espectro del sistema de titania-alúmina sintetizados por el método sol-gel en el rango UV-Vis, barrido en un intervalo de 200 a $700 \mathrm{~nm}$. 


\section{Difracción de rayos $X(D R X)$}

La caracterización por DRX mostró los patrones de difracción bien definidos y de mediana intensidad, dichos resultados se pueden observar en la Figura 5. El difractograma de la titania experimental tiene una congruencia aceptable con la carta 00-002-0406. De igual manera, la alúmina experimental concuerda con la carta 00-002-1420 [6].

Se observan los planos (101), (004), (002), (112), (200), (105), (211), (204), (116), (220), (215) y (224) que evidencian la forma anatasa correspondiente a la titania. Para la alúmina, se presentan los planos de difracción (220), (311), (400) y (844) que conforman la celda unitaria cúbica simple. Y los planos (220), (400) de la y-alúmina. Estas formas cristalinas presentan las mejores propiedades fotocatalíticas [6].

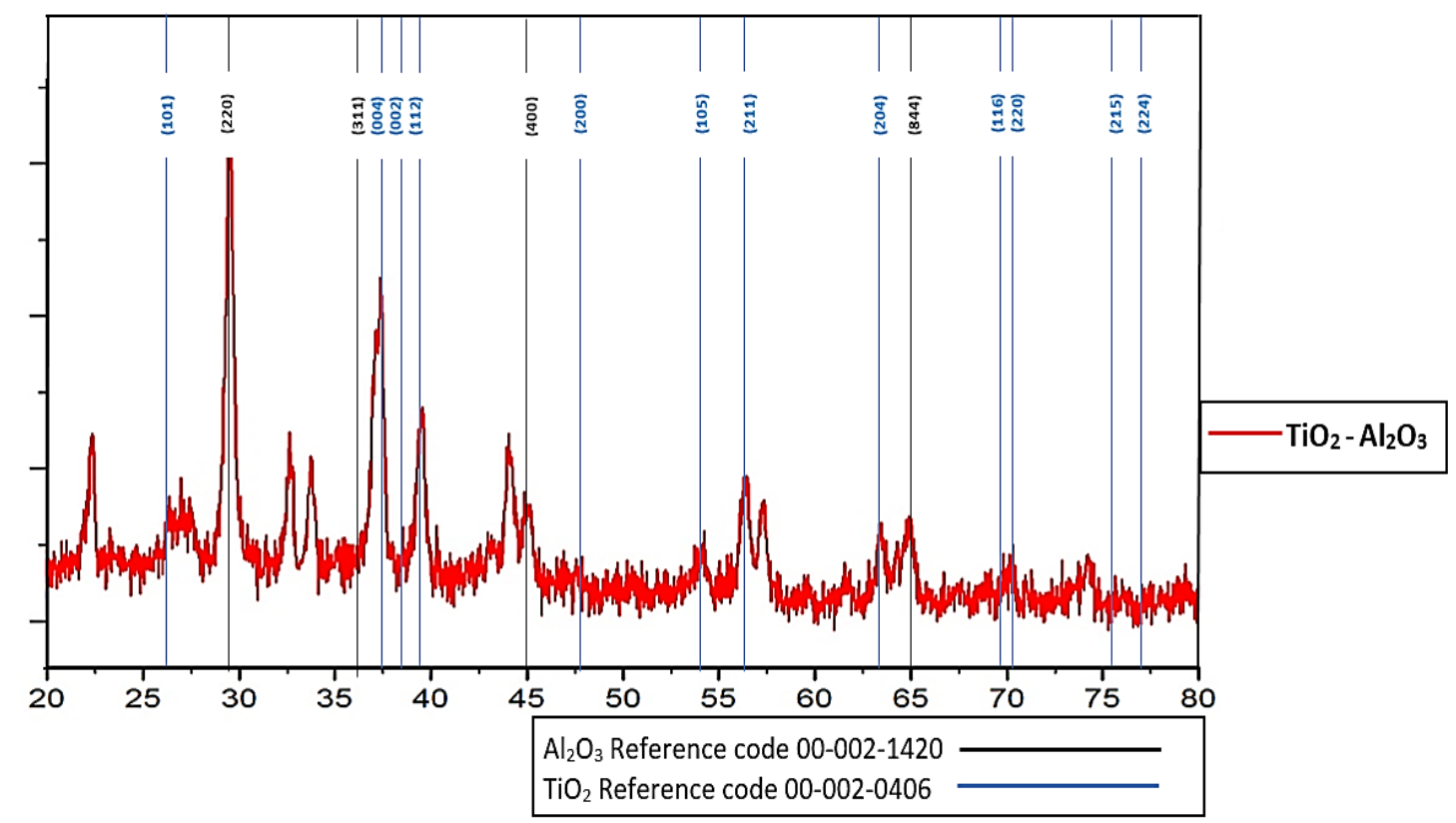

Figura 5. Difractograma del sistema de titania-alúmina sintetizados por el método sol-gel, [Elaboración propia].

\section{Microscopía Electrónica de Barrido (MEB)}

Las películas de $\mathrm{TiO}_{2}-\mathrm{Al}_{2} \mathrm{O}_{3}$, se caracterizaron después del octavo ciclo de pruebas fotocatalíticas. La Figura 6 muestra una topografía superficial de un sustrato recubierto con la película de $\mathrm{TiO}_{2}-\mathrm{Al}_{2} \mathrm{O}_{3}$, se observan partículas cuasi-esféricas de tamaño del orden de 30-40 nm. Las micrografías muestran una película distribuida uniformemente en el sustrato. No se presentan fracturas, lo que favorece a las condiciones de síntesis y a la técnica de recubrimiento por inmersión (dip-coating revolver) [6]. 


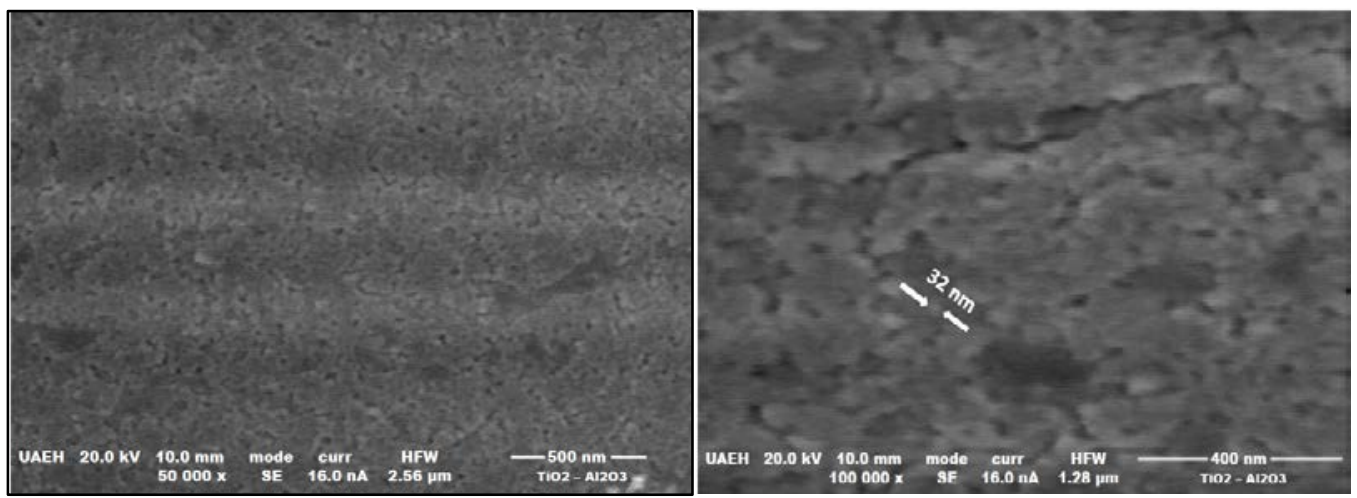

Figura 6. Micrografías del sistema de titania-alúmina sintetizados por el método sol-gel después de ocho procesos de fotocatálisis, lado izquierdo 50000 x, lado derecho $100000 x$

Degradación fotocatalítica de fenol y azul de metileno

La Figura 7 presenta el promedio de degradación fotocatalítica de 4 experimentaciones con fenol y 4 con azul de metileno como contaminantes orgánicos al interior del reactor y en contacto directo con las películas fotocatalíticas. Mostrando una eficiencia de degradación del $98.12 \%$ durante 6 h y $97.43 \%$ por 5 h respectivamente. Además de dos ensayos extras con cada contaminante para observar el comportamiento sin películas. En la Figura 8 se muestra efecto principal en el porcentaje de degradación de contaminación orgánica con respecto al tiempo.
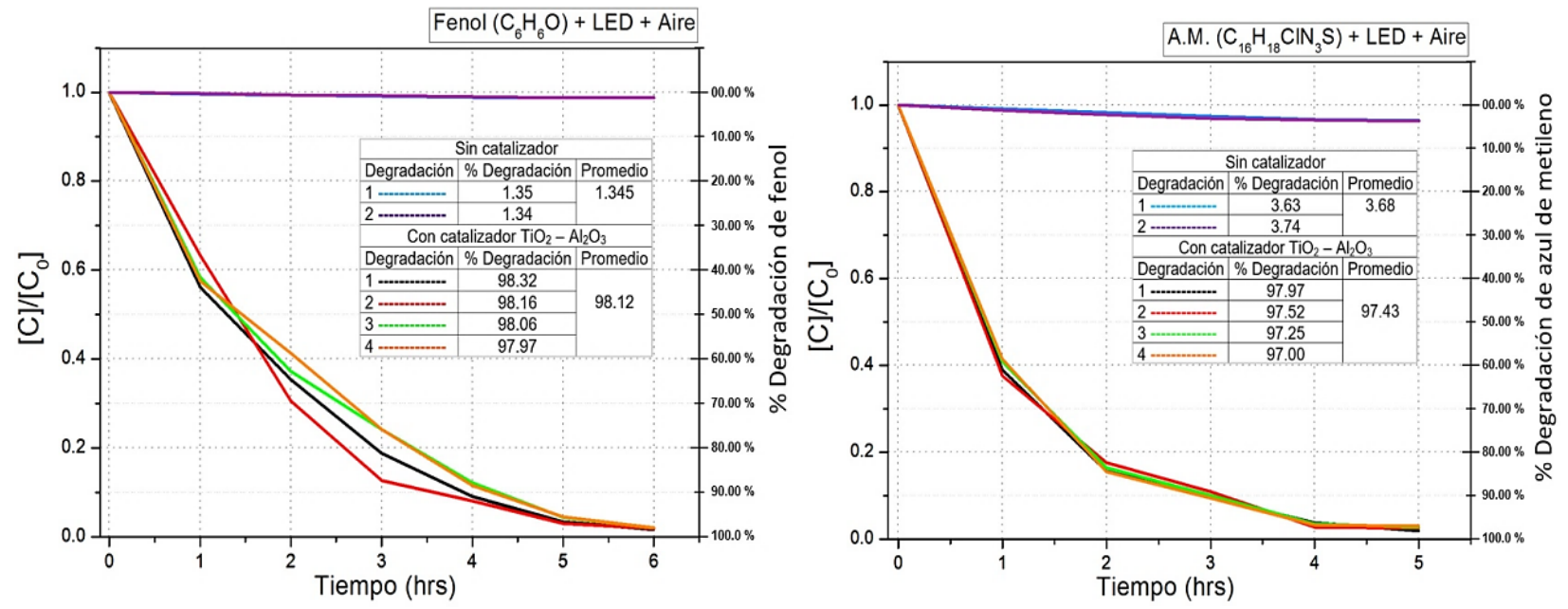

Figura 7. Gráficas comparativas del efecto principal en la concentración y en el porcentaje de degradación de fenol y azul de metileno con respecto al tiempo, con y sin película catalizadora de $\mathrm{TiO}_{2}-\mathrm{Al}_{2} \mathrm{O}_{3}$. 


\section{Cuantificación de Carbono Orgánico Total (COT)}

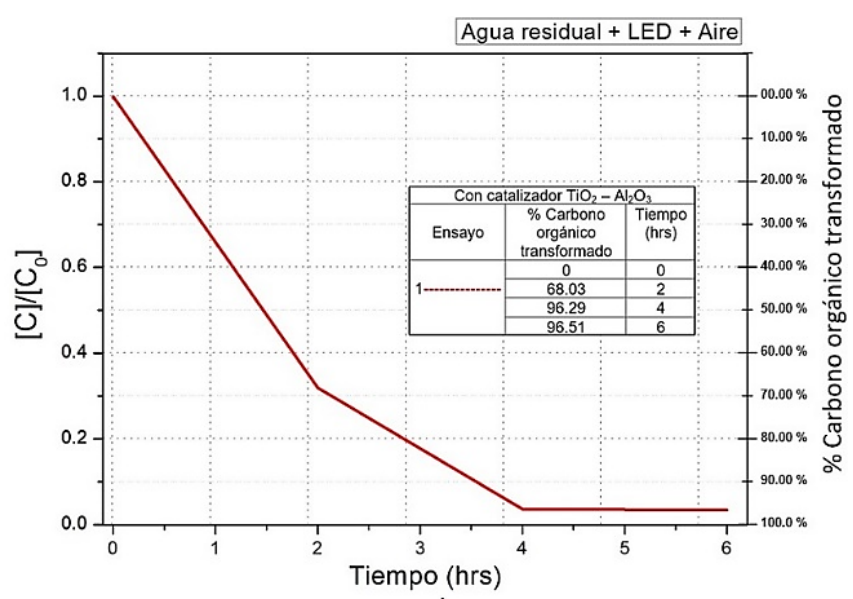

a)

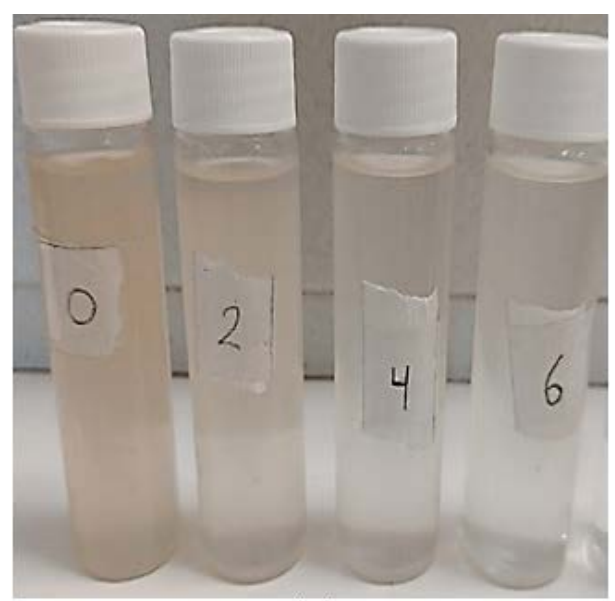

b)

Figura 8. a) Gráfica del efecto principal en el porcentaje de degradación de contaminación orgánica con respecto al tiempo, con película catalizadora de $\mathrm{TiO}_{2}-\mathrm{Al}_{2} \mathrm{O}_{3}$. b) Muestras recolectadas cada dos horas durante la reacción al interior del reactor [Elaboración propia].

La norma ISO 8245:1999 proporciona orientación para la determinación de carbono orgánico total (COT) en aguas residuales, es aplicable para muestras de agua con contenido de carbono orgánico que va desde $0.3 \mathrm{mg} / \mathrm{L}$ hasta $1000 \mathrm{mg} / \mathrm{L}$ [7].

En este caso se obtuvo un 96.51 \% de eficiencia de oxidación de carbono orgánico total durante seis horas, dejando solo un 3.49 \% de carbono orgánico, lo que representa una concentración final de $35.6 \mathrm{mg} / \mathrm{L}$. En la actualidad las EDAR tardan de 12 a 24 horas por ciclo de depuración, obteniendo agua cuya concentración final es de 64.9 a 134.5 mg/L.

\section{CONCLUSIONES}

Las condiciones de síntesis fueron satisfactorias para la obtención de películas del sistema mixto de $\mathrm{TiO}_{2}-\mathrm{Al}_{2} \mathrm{O}_{3}$, la presencia de las formas cristalinas individuales de los óxidos metálicos, anatasa del $\mathrm{TiO}_{2}$ y cúbica simple correspondiente a la ${ }_{\mathrm{\gamma}}-\mathrm{Al}_{2} \mathrm{O}_{3}$, indicó que hay una interacción molecular entre ambos óxidos, favoreciendo la dispersión de los dominios fotocatalíticos del $\mathrm{TiO}_{2}$, haciendo posible la activación del fotocatalizador en el espectro visible. La topografía superficial mostró homogeneidad de las películas con partículas cuasi-esféricas de tamaño del orden de 30-40 nm. La eficiencia fotocatalítica de las películas $\mathrm{TiO}_{2}-\mathrm{Al}_{2} \mathrm{O}_{3}$, fue en promedio del $97 \%$ para el caso de ambos contaminantes control (fenol y azul de metileno) empleando luz blanca artificial, despreciando el bajo porcentaje de fotólisis demostrado experimentalmente. De igual manera el análisis para la determinación de carbono orgánico total, demostró una oxidación casi total de una mezcla de contaminantes orgánicos complejos con una eficiencia fotocatalítica del $96.51 \%$, lo que representa una concentración final de 35.6 $\mathrm{mg} / \mathrm{L}$ obtenida en un periodo de seis horas, estando dentro de la normatividad vigente y obteniendo resultados favorables en comparación con los de una EDAR tradicional. Cabe mencionar que después de los tiempos de reacción ya mencionados, la concentración se mantiene constante y ya no disminuye en ningún contaminante. 


\section{AGRADECIMIENTOS}

A la maestra Mónica Araceli Camacho González, La Universidad Tecnológica de Tecámac, UTTEC y la Universidad Autónoma del Estado de Hidalgo, UAEH por su colaboración con equipos necesarios para los análisis y caracterizaciones respectivas, así como en el suministro de algunos materiales y reactivos.

\section{BIBLIOGRAFÍA}

[1] A. Mejía, M. Pérez. «Obtención y caracterización de óxido de titanio dopado con nitrógeno como fotocatalizador por le método de Pechini para uso en reactor solar (CPC).» San Luis Potosi: IPICyT (2014) 56-89.

[2] B. Wawrzyniak. Science 2016 (2016) 14-36.

[3] B. Bayarri, J. Giménez. Ciencia UANL. (2005) 227-236.

[4] B. Cavicchioli. American Journal of science. 2013 (2013) 12-35.

[5] E. Mecatalf. «Ingeniería de aguas residuales: tratamiento, vertido y reutilización. Tercera edición. McGraw-Hill. México (2012) 235-280.

[6] S. Malato, J. Blanco. Science 2019 (2019) 1-15.

[7] Yue, P. CENID 2013 (2013) 23-49. 